\title{
On Multipower Equations: Some Iterative Solutions and Applications
}

\author{
David K. Ruch and Patrick J. Van Fleet
}

\begin{abstract}
A generalization of McFarland's iterative scheme [12] for solving quadratic equations in Banach spaces is reported. The notion of a uniformly contractive system is introduced and subsequently employed to investigate the convergence of a new iterative method for approximating solutions to this wider class of multipower equations. Existence and uniqueness of solutions are addressed within the framework of a uniformly contractive system. To illustrate the usc of the new iterative scheme, we employ it when approximating solutions to a Hammerstein equation and a Chandrashekar equation. Due to the nature of the examples, we have found that wavelet/scaling function bases are a natural choice for the implementation of our iterative method.
\end{abstract}

Keywords: Multipower equations, k-linear equations, uniformly contractive systems, Hammerstein equations, Chandrashekar equations, wavelets

AMS subject classification: $47 \mathrm{H} 17,46 \mathrm{~N} 20,47 \mathrm{~N} 20,65 \mathrm{~J} 15$

\section{Introduction}

We seek the solution $x \in X$, where $X$ is a Banach space to the multipower equation

$$
y=x+\lambda L(\underbrace{x, \ldots, x}_{k \text { times }})
$$

Here, $y \in X$ and $L: X^{k} \mapsto X$ is a $k$-linear operator. The norm of $L$ is given in the usual way:

$$
\|L\|=\sup _{\left\|x_{i}\right\|<1}\left\|L\left(x_{1}, \ldots, x_{k}\right)\right\| .
$$

We shall consider for $k \geq 2$ the linear map

$$
L_{x}(\cdot):=L(\underbrace{x, \ldots, x}_{k-1 \text { times }}, \cdot)
$$

David K. Ruch: Sam Houston State University, Dep. Math., Huntsville, TX 77341 USA; email: mth_dkr@shsu.edu

Patrick J. Van Fleet: Sam Houston State University, Dep. Math., Huntsville, TX 77341 USA; e-mail: vanfleet@guy.shsu.edu

Research partially supported by SHSU Faculty Enhancement Award.

ISSN 0232-2064 / \$2.50 C Heldermann Verlag 
and observe that

$$
\left\|L_{x}\right\| \leq\|L\|\|x\|^{k-1}
$$

The following lemma gives a bound that is useful when measuring the effectiveness of our iterative scheme. The proof of the lemma is similar to that of Theorem 7.3 in [15], and is thus omitted.

Lemma 1.1. Let $L: X^{k} \mapsto X$ be a $k$-linear operator with $k \geq 2$. For $w, z \in X$ with $\|w\|,\|z\| \leq M$ we have

$$
\left\|L_{w}-L_{z}\right\| \leq\left(\|L\| M^{k-2}(k-1)\right)\|w-z\| .
$$

McFarland [12] considered $k=2$ in (1) and derived an iterative scheme for approximating solutions to quadratic equations in Banach spaces. He obtains convergence results for his method by using a continued fraction approach. In order to generalize his method to arbitrary $k$, we have employed the contractive mapping theorem. We give conditions on the operators involved and the initial guesses that will guarantee convergence of the iterative scheme. The use of the contractive mapping theorem yields a uniqueness of solution result as well.

We then show that these results can be generalized to a wider class of equations where the operator $L$ need not be linear in the first $k-1$ variables, and an application to boundary value problems is given. We conclude Section 2 with a uniqueness result for "small" solutions of equation (1). lem

In order to approximate solutions to equation (1), we consider the fixed point prob-

$$
Q(x)=x,
$$

where, for appropriate $\lambda \in \mathbb{R}$ and $x \in X$, the map $Q: X \mapsto X$ is given as follows:

$$
Q(x)=\left(\lambda L_{x}+I\right)^{-1}(y) .
$$

The difficulty lies with inverting an infinite dimensional linear operator, so the standard approach is to use successive subspaces $V_{n}$ and approximate the solution to the problem in finite-dimensional settings. Uniformly contractive systems will be developed to show that these finite-dimensional approximations do indeed converge to the true solution of equation (4).

To formulate the finite-dimensional approximating scheme, we first assume that $X$ has a Schauder basis $\left\{e_{k}\right\}_{k=1}^{\infty} \subset X$. Then each $x \in X$ has a unique representation

$$
x=\sum_{k=1}^{\infty}\left\langle e_{k}^{\prime}, x\right\rangle e_{k},
$$

where $e_{k}^{\prime} \in X^{\prime}$ satisfy $\left\langle e_{k}^{\prime}, e_{j}\right\rangle=\delta_{k j}$.

Next, let $\left\{k_{n}\right\} \subset \mathbb{N}$ be an increasing sequence. We then define the projection operators $S_{n}$ as follows:

$$
S_{n}(x)=\sum_{j=1}^{k_{n}}\left\langle e_{j}^{\prime}, x\right\rangle e_{j}
$$


Since $X$ is complete, $\sup _{n \in N}\left\|S_{n}\right\|<\infty$. For convenience, we assume that $\left\|S_{n}\right\|=1$. We then take as our finite-dimensional subspaces $V_{n}=S_{n}(X)$, and define the linear $\operatorname{map} L_{x}^{n}: X \mapsto V_{n}$ as

$$
L_{x}^{n}(\cdot)=S_{n}\left(L_{x}(\cdot)\right)
$$

We recall that a $k$-linear operator $L: X^{k} \mapsto X$ is compact if for any bounded set $B \subset X$, the set $L\left(B^{k}\right)$ is relatively compact. For more details on compact multilinear maps, please see $[14,18,20]$.

The next result illustrates that compactness is sufficient to ensure that $L_{x}^{n} \rightarrow L_{x}$.

Lemma 1.2. Suppose that $X$ has a Schauder basis.

(i) If $L$ is compact in its $k$ th variable, then for each $x \in X$,

$$
\lim _{n \rightarrow \infty}\left\|L_{x}^{n}-L_{x}\right\|=0
$$

(ii) If $L$ is compact, then

$$
\lim _{n \rightarrow \infty}\left\|L^{n}-L\right\|=0
$$

Here, $L^{n}=S_{n} L$.

Proof. It is clear that $S_{n}$ converges uniformly to the identity map $I$ on relatively compact sets. Since $L_{x}$ is a compact map, for any bounded set $B \subset X$, the set $L_{x}(B)$ is relatively compact. Now $L_{\boldsymbol{x}}$ is linear, so

$$
\left\|L_{x}^{n}-L_{x}\right\|=\left\|\left(S_{n}-I\right) L_{x}\right\|=\sup _{\|w\| \leq 1}\left\|\left(S_{n}-I\right) L_{x}(w)\right\| \rightarrow 0
$$

which proves assertion (i).

If $L$ is compact, then $L\left(B^{k}\right)$ is relatively compact for any bounded set $B \subset X$, so

$$
\lim _{n \rightarrow \infty}\left\|L^{n}-L\right\|=\lim _{n \rightarrow \infty}\left\|\left(S_{n}-I\right) L\right\|=0
$$

which is assertion (ii)

The preceeding result will be used in Section 4 when we derive conditions that guarantee convergence of the sequence of solutions obtained in finite-dimensional subspaces to the true fixed point solution of equation (4). Sufficient conditions on the operator $L$ for this convergence compare favorably with those given in [1].

In order to obtain the results of Section 4, we have found it convenient to develop the notion of a uniformly contractive system. Such a system is defined in Section 3 and is a useful framework with which to show that $z_{n} \rightarrow z$, where $z$ solves equation (4) and the $z_{n}$ are the fixed points of the $\operatorname{map} Q_{n}: X \rightarrow V_{n}$. Uniformly contractive systems have also been used in [19] with another iterative scheme for solving certain nonlinear operator equations.

We conclude the paper with a section of examples illustrating the application of our iterative scheme to approximating solutions of certain multipower equations. We consider approximating solutions to the 
(i) Hammerstein equation

$$
y(s)=x(s)+\lambda \int_{a}^{b} K(s, t)(x(t))^{2} d t
$$

where $X=L^{2}[a, b]$ and the

(ii) Chandrashekar equation

$$
1=H(s)+\frac{1}{2} \lambda s H(s) \int_{0}^{1} \frac{H(t)}{s+t} d t
$$

where $X=L^{2}[0,1]$.

In both cases, we use a sequence of closed nested subspaces $V_{0} \subset \ldots \subset V_{n} \subset X$. Such sequences of subspaces have been found to be particularly useful in many applications when the $V_{n}$ form a so-called multiresolution analysis (see $[7,13]$ and references therein). We may then employ a sequence of wavelet bases $\left\{\psi_{n k}\right\} \subset V_{n}$ for providing approximate solutions to the multipower equations. These bases are orthonormal and compactly supported. Such properties are desirable in view of the number of integrals that must be computed when we devise a scheme for obtaining approximate solutions to equations (i) and (ii) above. A discussion of the algorithm used for obtaining approximate solutions is also included into this section.

\section{Multipower operator equations}

We begin with a lemma that will be of use later in this section.

Lemma 2.1. For $k \geq 2$ and $0<\alpha<\frac{(k-1)^{k-1}}{k^{k}}$, there exists some $D>0$ such that

$$
\frac{\alpha(1+D)^{k-1}}{1-\alpha(1+D)^{k-1}}<D<\frac{1}{k-1} .
$$

Proof. Observe that the left inequality in (12) is equivalent to

$$
\alpha(1+D)^{k}-(1+D)+1<0 .
$$

Now let $f(v)=\alpha v^{k}-v+1$. Clearly $f(1)>0$ and since $\alpha<\frac{(k-1)^{k-1}}{k^{k}}$, we find that $f\left(\frac{k}{k-1}\right)<0$. Thus there exists some $v=1+D$ satisfying $1<v<\frac{k}{k-1}$ so that the desired inequality (12) holds scheme

The following theorem gives conditions on $\lambda, L$, and $y$ to ensure that the iteration

$$
x_{n+1}=Q\left(x_{n}\right)
$$

converges to the true fixed point of (4). Such conditions also lead to the definition of a sphere $S$ wherein any initial guess $x_{0}$ will lead to the unique fixed point in some sphere $U \subset S$. 
Theorem 2.2. If

$$
0<|\lambda|\|L\|\|y\|^{k-1}=\alpha<\frac{(k-1)^{k-1}}{k^{k}}
$$

then there exists a solution $x$, to equation (1), unique in the open sphere

$$
S=\left\{z \in X \mid\|z-y\|<\frac{\|y\|}{k-1}\right\} \text {. }
$$

If the initial guess $x_{0} \in S$, then

$$
\lim _{n \rightarrow \infty} x_{n}=x,
$$

where $\dot{x}_{n+1}=Q\left(x_{n}\right)$. The solution $x$, is contained in the closed sphere

$$
U=\left\{z \in X \mid\|z-y\| \leq D\|y\|<\frac{\|y\|}{k-1}\right\}
$$

where $D$ is given in Lemma 2.1 .

Proof. Let $x_{0} \in S$ and set $\alpha=|\lambda|\|L\|\|y\|^{k-1}$. By Lemma 2.1, we can choose $D$ so that

$$
\frac{\left\|x_{0}-y\right\|}{\|y\|}<D<\frac{1}{k-1} \quad \text { and } \quad \frac{\alpha(1+D)^{k-1}}{1-\alpha(1+D)^{k-1}}<D \text {. }
$$

Note that $x_{0}$ is in the closed sphere $U$. We claim that $Q(U) \subset U$. To verify the claim, let $x \in U$. Then

$$
Q(x)-y=\left(\lambda L_{x}+I\right)^{-1}\left(I-\left(\lambda L_{x}+I\right)\right)(y)
$$

so that

$$
\begin{aligned}
\|Q(x)-y\| & \leq \frac{\left\|\lambda L_{x}\right\|}{1-\left\|\lambda L_{x}\right\|}\|y\| \\
& \leq \frac{\|\lambda L\|((1+D)\|y\|)^{k-1}}{1-\|\lambda L\|((1+D)\|y\|)^{k-1}}\|y\| \\
& =\frac{\alpha(1+D)^{k-1}}{1-\alpha(1+D)^{k-1}}\|y\| \\
& \leq D\|y\| .
\end{aligned}
$$

Thus $Q(x) \in U$, which proves the claim.

Next we show that $Q: U \mapsto U$ is a contraction mapping, with contraction factor

$$
r=\frac{\alpha(1+D)^{k-2}(k-1)}{\left(1-\alpha(1+D)^{k-1}\right)^{2}}<1
$$

(to see that $r<1$, note that replacing $D$ with $\frac{1}{k-1}$ and $\alpha$ with $\frac{(k-1)^{k-1}}{k^{k}}$ yields $r=1$ ). In order to prove that $Q$ is indeed a contraction, let $x, w \in U$. Then

$$
Q(x)-Q(w)=\left(\lambda L_{x}+I\right)^{-1}\left(\lambda L_{w}-\lambda L_{x}\right)\left(\lambda L_{w}+I\right)^{-1} y
$$


which along with Lemma 1.1 and repeated use of (2) yields

$$
\begin{aligned}
\|Q(x)-Q(w)\| & \leq \frac{\|\lambda L\|\left(\|y\|^{k-2}(1+D)^{k-2}(k-1)\right)\|w-x\|}{\left(1-\left\|\lambda L_{x}\right\|\right)\left(1-\left\|\lambda L_{w}\right\|\right)}\|y\| \\
& \leq \frac{\alpha(1+D)^{k-2}(k-1)\|w-x\|}{\left(1-\|\lambda L\|((1+D)\|y\|)^{k-1}\right)^{2}} \\
& =r\|w-x\| .
\end{aligned}
$$

Now since $Q$ is a contraction mapping with $Q(U) \subset U$, we can apply the contraction mapping principle to the iterative scheme $x_{n+1}=Q\left(x_{n}\right)$ and conclude that the iterates must converge to the unique fixed point $x_{s} \in U$ of $Q$. Since $D$ can be chosen arbitrarily close to $\frac{1}{k-1}$, the solution must be unique in the open sphere $S$

Corollary 2.3. The bound $\frac{(k-1)^{k-1}}{k^{k}}$ on $|\lambda|\|L\|\|y\|$ in (14) is optimal for $X=\mathbb{R}$.

Proof. Consider the equation $1=x-\frac{(k-1)^{k-1}}{k^{k}} x^{k}$

Some remarks are in order before we conclude this section. We first note that the iterative method described in Theorem 2.2 can be generalized slightly to solve equations of the form

$$
y=A x+\lambda L(x, \ldots x)
$$

where $A$ and $A^{-1}$ are linear and bounded. Putting $y^{a}=A^{-1} y$ and $L^{a}=A^{-1} L$, we have

$$
y^{a}=x+\lambda L^{a}(x, \ldots x)
$$

which is of the form (1) and can thus be solved using Theorem 2.2.

From Lemma 2.1, we observe that for $\lambda$ near 0 , we can pick $D$ near 0 and conclude that the solution to equation (1) is close to $y$, thus improving our choice of the initial guess in the iterative scheme (13).

In the special case $k=2$, it is useful to compare our results with those obtained elsewhere. In [12], McFarland considered the equation (18) with $A$ invertible and linear. McFarland showed in his Theorem 3 that the iterative scheme

$$
x_{n+1}=\left(A+\lambda L x_{n}\right)^{-1} y=\left(I+\lambda L^{a} x_{n}\right)^{-1} y^{a}
$$

converges to a solution of equation (18) if

$$
0<\left\|A^{-1}\right\||\lambda|\|L\|\left\|y^{a}\right\| \leq \delta<\frac{1}{4}
$$

and if

$$
\frac{1-(1-4 \delta)^{1 / 2}}{2} \leq\left\|\lambda L^{a} x_{0}\right\|<\frac{1}{2}
$$

If our condition (14) with $k=2$ is satisfied, then so is McFarland's condition (19). Note that McFarland's requirement (20) on the initial condition $x_{0}$ may be more difficult to verify than the condition (15) $\left\|x_{0}-y\right\|<\|y\|$. McFarland does not use the contraction mapping principle in his proof, and obtains no uniqueness results. 
In [17], Rall solves equation (1) subject to (14), both with $k=2$. He uses a series approach and shows in his Theorem 19 that the solution $x$ is unique in the sphere

$$
\left\{z \in X \mid\|z-x\|<\frac{\sqrt{1-4|\lambda|\|L\|\|y\|}}{2|\lambda|\|L\|}\right\} \text {. }
$$

When $4|\lambda|\|L\|\|y\|$ is near 1, Rall's uniqueness condition (21) does not give as much information as our condition (15). On the other hand, for $\lambda$ near 0 , Rall's sphere is much larger than our sphere $S$ in (15).

In [1], Argyros uses a different iterative method and an auxiliary quadratic equation to obtain several existence and uniqueness results for equation (1) when $k=2$. We state a corollary of Argyros [1] for the purpose of comparison to our results.

Corollary 2.4 (Argyros [1]). For any $y \in X$ such that $\|y\|<4(|\lambda|\|L\|)^{-1}$,

(i) equation (1) has a unique solution $x \in U(y, a)$, where

$$
a=(1-2|\lambda|\|L\|\|y\|)(2|\lambda|\|L\|)^{-1} ;
$$

(ii) moreover $x \in \bar{U}(y, b)$, where

$$
b=\left(1-2|\lambda|\|L\|\|y\|-(1-4|\lambda|\|L\|\|y\|)^{1 / 2}\right)(2|\lambda|\|L\|)^{-1} .
$$

Argyros' uniqueness ball (i) is bigger than ours (15) but his existence ball (ii) is the same as ours. We summarize this in the following corollary.

Corollary 2.5. In the case $k=2$, the solution $x_{s}$ of equation (1) is in the closed sphere

$$
\begin{aligned}
U & =\{z \in X \mid\|z-y\| \leq \tilde{D}\|y\|\} \\
& =\left\{z \in X \mid\|z-y\| \leq \frac{1-2|\lambda|\|L\|\|y\|-(1-4|\lambda|\|L\|\|y\|)^{1 / 2}}{2|\lambda|\|L\|}\right\} .
\end{aligned}
$$

Proof. It is easy to see that there exists a unique root $v_{0} \in\left(1, \frac{k}{k-1}\right)$ for the function $f(v)$ given in the proof of Lemma 2.1. Then for each $v \in\left(v_{0}, \frac{k}{k-1}\right), D=v-1$ satisfies inequality (12) and so by Theorem 2.2

$$
\left\|y-x_{s}\right\| \leq D\|y\|=(v-1)\|y\| .
$$

Hence $\left\|y-x_{s}\right\| \leq\left(v_{0}-1\right)\|y\|$. In the case $k=2$, the quadratic formula yields

$$
\left(v_{0}-1\right)\|y\| \leq \frac{1-2|\lambda|\|L\|\|y\|-(1-4|\lambda|\|L\|\|y\|)^{1 / 2}}{2|\lambda|\|L\|}
$$

and the assertion is proved

The linearity of $L$ in the first $k-1$ variables is not critical for the results of this section, and we can generalize them somewhat. 
Proposition 2.6. Let $L: X \times X \rightarrow X$ satisfy the following conditions:

(i) $L_{x}:=L(x, \cdot)$ is a bounded linear operator for all $x \in X$.

(ii) There exist $\mu \geq 2$ and $C>0$ such that

$$
\|L(x, v)\| \leq C\|x\|^{\mu-1}\|v\|
$$

and

$$
\left\|L_{x}-L_{v}\right\| \leq C(\mu-1)(\max (\|x\|,\|v\|))^{\mu-2}\|x-v\|
$$

for all $x, v \in X$.

Then Theorem 2.2 and Corollary 2.5 hold for this operator $L$ with $\|L\|$ and $k$ replaced by $C$ and $\mu$, respectively, throughout the theorem and corollary.

Proof. Note that condition (ii) is just a minor generalization of inequalities (2) and (3). The proofs of Theorem 2.2 and Corollary 2.5 are valid with these adjustments

It is clear from Lemma 1.1 that a bounded $k$-linear operator will satisfy the hypotheses of this proposition. The next example is an important example of an operator of this type that is not $k$-linear.

Example 2.7. Define $L: C[0,1] \times C[0,1] \rightarrow C[0,1]$ by

$$
L(x, v)(s)=\int_{0}^{1} h(s, t)(x(t))^{\mu-1} v(t) d t
$$

where $h \in C\left([0,1]^{2}\right)$ and $\mu \geq 2$. Then $L$ is linear in $v$ and

$$
\|L(x, v)\|_{\infty} \leq\|x\|_{\infty}^{\mu-1}\|v\|_{\infty}\left\|\int_{0}^{1} h(s, t) d t\right\|_{\infty} .
$$

Moreover,

$$
\begin{aligned}
\left\|\left(L_{x}-L_{w}\right)(v)\right\|_{\infty} & =\left\|\int_{0}^{1} h(s, t)\left((x(t))^{\mu-1}-(w(t))^{\mu-1}\right) v(t) d t\right\|_{\infty} \\
& \leq(\mu-1) M^{\mu-2}\left\|\int_{0}^{1} h(s, t) d t\right\|_{\infty}\|x-w\|_{\infty}\|v\|_{\infty},
\end{aligned}
$$

where $M=\max \left(\|x\|_{\infty},\|w\|_{\infty}\right)$, by the lemma below.

Lemma 2.8. For $x, w \geq 0$ and $\mu \geq 2$,

$$
\left|x^{\mu-1}-w^{\mu-1}\right| \leq(\mu-1)(\max (x, \mu))^{\mu-2}|x-w| \text {. }
$$

Proof. Without loss of generality, assume $w<x$. Fix $w$ and let

$$
f(x)=(\mu-1) x^{\mu-2}(x-w) \quad \text { and } \quad g(x)=x^{\mu-1}-w^{\mu-1} .
$$

Then

$$
f^{\prime}(x)=(\mu-1) x^{\mu-2}+(x-w)(\mu-1)(\mu-2) x^{\mu-3} \geq(\mu-1) x^{\mu-2}=g^{\prime}(x) .
$$

Since $f(w)=0=g(w)$, inequality (23) yields (22) 
Remark. The class of boundary value problems (se, for example, $[10,11]$ )

$$
x^{\prime \prime}(t)+\lambda a(t) x^{\mu}(t)=f(t)
$$

with $t \in[0,1], \mu \geq 2$ and appropriate boundary conditions can be transformed into

$$
x(t)=\lambda \int_{0}^{1} K(s, t) a(s) x^{\mu}(s) d s+F(t)
$$

Then Proposition 2.6 will apply with suitable restrictions on $\lambda$ and $a(t)$.

For the case $y=0$ in equation (1), our iterative scheme

$$
x_{n}=\left(\lambda L_{x_{n}}+I\right)^{-1}(y)
$$

will yield only the trivial solution $x=0$. We show below that this is the only "small" solution. To obtain "large" solutions, schemes such as the Newton-Kantorovich method [2] can be used. For many problems, the Newton-Kantorovich method will be faster than our iterative scheme. However, if the Fréchet derivative $\lambda L^{\prime}(x, \ldots, x)$ is not defined or is significantly more expensive to numerically compute than $\lambda L_{x}$, then our scheme is preferable.

Proposition 2.9. Equation (1) has at most one solution $x \in X$ for which

$$
\|x\|<\left(\frac{1}{k\|L\|}\right)^{\frac{1}{k-1}} .
$$

Proof. For the sake of reaching a contradiction, suppose that $u$ and $v$ are distinct solutions to equation (1) satisfying (24). Then

$$
\begin{aligned}
u-v & =L(u, \ldots, u)-L(v, \ldots, v) \\
& =L(u-v, u, \ldots, u)+L(v, u-v, u, \ldots, u)+\ldots+L(v, \ldots, v, u-v)
\end{aligned} .
$$

so by (24) we have

$$
\|u-v\| \leq k\|u-v\|\|L\|(\max (\|u\|,\|v\|))^{k-1}<\|u-v\|
$$

which is a contradiction 


\section{Uniformly contractive systems}

We now introduce the notion of a uniformly contractive system. The role of such system is to provide a general framework for obtaining iterative solutions of operator equations that involve contraction mappings. In particular, we will use the concept of the uniformly contractive sytem in conjunction with the method discussed in Section 2 to construct approximate solutions to certain multipower equations.

Definition 3.1. Let $X$ be a Banach space, $\left\{V_{n}\right\}$ a sequence of closed subspaces of $X$ such that

$$
\lim _{n \rightarrow \infty} \operatorname{dist}\left(V_{n}, x\right)=0
$$

for each $x \in X$. Let $U$ be a closed set in $X$, and, for each $n \in \mathbb{N}$, let $Q_{n}$ be an operator with $Q_{n}: X \mapsto V_{n}$, and define the set $U_{n}=V_{n} \cap U$. We say that $\left\{U_{n}, Q_{n}\right\}$ is a uniformly contractive system if conditions (C1) and (C2) below hold.

(C1) There exists a $c \in \mathbb{R}, 0<c<1$, and an $N \in \mathbb{N}$ such that if $n \geq N$ and $x, y \in U$, then

$$
Q_{n}(U) \subset U_{n} \quad \text { and } \quad\left\|Q_{n}(x)-Q_{n}(y)\right\| \leq c\|x-y\| .
$$

(C2) For any $x, y \in U$ and $\varepsilon>0$, there exists an $N \in \mathbb{N}$ such that if $k \geq j \geq N$, then

$$
\left\|Q_{k}(x)-Q_{j}(y)\right\| \leq c\|x-y\|+\varepsilon .
$$

Note that the subspaces $V_{n}$ need not be nested, so that the finite element method can be used within the context of a uniformly contractive system.

Theorem 3.2. Let $\left\{U_{n}, Q_{n}\right\}$ satisfy condition (C1) above. Then condition (C2) is equivalent to the existence of a contraction map $Q: U \mapsto U$, defined by $Q(x)=$ $\lim _{n \rightarrow \infty} Q_{n}(x)$, such that

$$
\|Q(x)-Q(y)\| \leq c\|x-y\|
$$

for $x, y \in U$.

Proof. Assume condition (C2) holds. We first show that the map $Q$ is well defined. Fix $x \in U$ and $\varepsilon>0$. Choose $N$ as prescribed in condition (C2) and set $y=x$ in (26). Then for $k \geq j \geq N$ we have

$$
\left\|Q_{k}(x)-Q_{j}(x)\right\| \leq \varepsilon
$$

Thus $\left\{Q_{j}(x)\right\}_{j}$ is a Cauchy sequence. Since $X$ is complete, $\lim _{n \rightarrow \infty} Q_{n}(x)$ exists. Noting that $U$ is closed and $Q_{n}(x) \in U_{n} \subset U$ for all $n$ yields

$$
\lim _{n \rightarrow \infty} Q_{n}(x)=Q(x) \in U .
$$

Now let $x, y \in U$ and $\varepsilon>0$. Choose $N \in \mathbb{N}$ so that

$$
\left\|Q(x)-Q_{N}(x)\right\|<\varepsilon \quad \text { and } \quad\left\|Q(y)-Q_{N}(y)\right\|<\varepsilon .
$$

Then

$$
\begin{aligned}
\|Q(x)-Q(y)\| & \leq\left\|Q(x)-Q_{N}(x)\right\|+\left\|Q_{N}(x)-Q_{N}(y)\right\|+\left\|Q_{N}(y)-Q(y)\right\| \\
& \leq \varepsilon+c\|x-y\|+\varepsilon .
\end{aligned}
$$


Since $\varepsilon$ is arbitrary, we have

$$
\|Q(x)-Q(y)\| \leq c\|x-y\|
$$

Next we assume the existence of the map $Q$, and fix $x, y \in U$ and $\varepsilon>0$. By the definition of $Q$, there exists an $N \in \mathbb{N}$ such that if $k \geq j \geq N$, then

$$
\begin{aligned}
\left\|Q_{k}(x)-Q_{j}(y)\right\| & \leq\left\|Q_{k}(x)-Q(x)\right\|+\|Q(x)-Q(y)\|+\left\|Q(y)-Q_{j}(y)\right\| \\
& \leq \varepsilon+c\|x-y\|+\varepsilon .
\end{aligned}
$$

Thus condition (C2) is satisfied

We observe that the equations $Q_{n}(x)=x$ all have unique fixed points $z_{n} \in U$ by the contraction mapping principle (see [9]). Our next result shows that these fixed points converge to $z_{s}$, the unique fixed point of the map $Q \in U$

Theorem 3.3. Let $\left\{U_{n}, Q_{n}\right\}$ be a uniformly contractive system. Then

$$
\lim _{n \rightarrow \infty} z_{n}=z_{s}, \quad \text { where } Q\left(z_{s}\right)=z_{s} .
$$

Proof. Let $\varepsilon>0$ and $0<c<1$ be the contractive constant for $\left\{U_{n}, Q_{n}\right\}$. By Theorem 3.2, we can choose $N \in \mathbb{N}$ so that $n \geq N$ implies that

$$
\left\|Q\left(z_{s}\right)-Q_{n}\left(z_{s}\right)\right\|<(1-c) \varepsilon
$$

Then

$$
\begin{aligned}
\left\|z_{s}-z_{n}\right\| & =\left\|Q\left(z_{s}\right)-Q_{n}\left(z_{n}\right)\right\| \\
& \leq\left\|Q\left(z_{s}\right)-Q_{n}\left(z_{s}\right)\right\|+\left\|Q_{n}\left(z_{s}\right)-Q_{n}\left(z_{n}\right)\right\| \\
& <(1-c) \varepsilon+c\left\|z_{s}-z_{n}\right\| .
\end{aligned}
$$

So

$$
\left\|z_{9}-z_{n}\right\|(1-c)<(1-c) \varepsilon \quad \text { whence } \quad\left\|z_{s}-z_{n}\right\|<\varepsilon
$$

Thus $\lim _{n \rightarrow \infty} z_{n}=z$,

Theorem 3.4. Let $\left\{U_{n}, Q_{n}\right\}$ be a uniformly contractive system such that $U$ is bounded and $\left\{Q_{n}\right\}$ converges to $Q$ uniformly on $U$. Let $N \in \mathbb{N}$ be given as per condition (C1). Beginning with any $k \geq N$ and initial guess $x_{k} \in U_{k}$, the iterative scheme.

$$
x_{n+k+1}=Q_{n}\left(x_{n+k}\right)
$$

will converge to the fixed point of $Q$ in $U: \lim _{n \rightarrow \infty} x_{n+k}=z_{s}=Q\left(z_{s}\right)$

Remark. We note at this time that to numerically implement (29), a hierarchical basis, such as one provided by a multiresolution analysis, is required. 
Proof of Theorem 3.4. Fix $\varepsilon>0$. By the uniform convergence of $\left\{Q_{n}\right\}$, there is some $M_{1} \geq k \geq N$ such that for $n \geq m \geq M_{1}$, we have

$$
\begin{aligned}
\left\|x_{n}-x_{m}\right\| & =\left\|Q_{n-1}\left(x_{n-1}\right)-Q_{m-1}\left(x_{m-1}\right)\right\| \\
& \leq\left\|Q_{n-1}\left(x_{n-1}\right)-Q_{m-1}\left(x_{n-1}\right)\right\|+\left\|Q_{m-1}\left(x_{n-1}\right)-Q_{m-1}\left(x_{m-1}\right)\right\| \\
& \leq \varepsilon+c\left\|x_{n-1}-x_{m-1}\right\| .
\end{aligned}
$$

Now choose $M_{2}>M_{1}$ such that

$$
c^{M_{2}-M_{1}}<\frac{\varepsilon}{2 \operatorname{diam} U}
$$

Then for any $n \geq m \geq M_{2}$, we can repeat the iteration (30) $m-M_{1}$ times to obtain

$$
\begin{aligned}
\left\|x_{n}-x_{m}\right\| & \leq \varepsilon \sum_{j=0}^{m-M_{1}-1} c^{j}+c^{m-M_{1}}\left\|x_{n-\left(m-M_{1}\right)}-x_{M_{1}}\right\| \\
& \leq \varepsilon \sum_{j=0}^{m-M_{1}-1} c^{j}+2 c^{m-M_{1}} \operatorname{diam} U \\
& \leq \varepsilon\left(\sum_{j=0}^{m-M_{1}-1} c^{j}+1\right) .
\end{aligned}
$$

Thus $\left\{x_{n}\right\}$ is a Cauchy sequence, with $\lim _{n \rightarrow \infty} x_{n}=z \in U$.

Now $\left\{U_{n}, Q_{n}\right\}$ is a uniformly contractive system, so for $n \geq N$,

$$
\begin{aligned}
\|Q(z)-z\| & \leq\left\|Q(z)-Q_{n}(z)\right\|+\left\|Q_{n}(z)-Q_{n}\left(x_{n}\right)\right\|_{i}+\left\|Q_{n}\left(x_{n}\right)-z\right\| \\
& \leq\left\|Q(z)-Q_{n}(z)\right\|+c\left\|z-x_{n}\right\|+\left\|x_{n+1}-z\right\| .
\end{aligned}
$$

For $n$ sufficiently large, we have

$$
\|Q(z)-z\| \leq\left\|Q(z)-Q_{n}(z)\right\|+c\left\|z-x_{n}\right\|+\left\|x_{n+1}-z\right\| \leq \varepsilon
$$

since $\left\{Q_{n}\right\}$ converges to $Q$ pointwise and $\lim _{n \rightarrow \infty} x_{n}=z$. As $\varepsilon$ is arbitrary, we have $Q(z)=z$. Since $Q$ has a unique fixed point in $U$, we conclude that $z_{s}=z$

Remark. The convergence rate for the scheme (29) to the solution $z$ of the fixed point problem (4) will be governed by the size of the contraction constant $c$ of the uniformly contractive system, as well as the diameter of $U$ and the uniform convergence of the operators $Q_{n}$ on $U$. To be precise, for any given $\varepsilon>0$, there are $M_{1}, M_{2} \in \mathbb{N}$ such that

$$
\left\|Q_{n}(x)-Q_{m}(x)\right\|<\varepsilon
$$

for all $m, n \geq M_{2} \geq M_{1}$ and $x \in U$. Then

$$
\left\|z-x_{m}\right\| \leq \varepsilon\left(\sum_{j=0}^{M_{2}-M_{1}} c^{j}+1\right)
$$

for $m \geq M_{2}$. 


\section{Applications of uniformly contractive systems for $X$ possessing a Schauder basis}

Let $X$ be a Banach space with Schauder basis $\left\{e_{k}\right\} \subset X$. Consider the operator

$$
R=\left(\lambda L_{\mathbf{x}}^{\mathbf{n}}+I\right) S_{\mathrm{n}}
$$

as a map from $V_{n}$ into $V_{n}$. Note that $R^{-1}: V_{n} \mapsto V_{n}$ exists when $\left\|\lambda L_{x}^{n} S_{n}\right\| \leq\left\|\lambda L_{x}\right\|<1$. In matrix terms, with respect to the basis $\left\{e_{k}\right\}, R^{-1}$ is formed by inverting the principal submatrix (corresponding to $V_{n}$ ) of the matrix representation of the linear operator $\left(\lambda L_{x}^{n}+I\right) S_{n}$. $X$ by

Let $J_{n}: V_{n} \mapsto X$ denote the natural injection operator. Define $\left(\lambda L_{x}^{n}+S_{n}\right)^{-1}: X \mapsto$

$$
\left(\lambda L_{x}^{n}+S_{n}\right)^{-1}=J_{n} R^{-1} S_{n}
$$

and define $Q_{n}: X \rightarrow V_{n}$ to be

$$
Q_{n}(x)=\left(\lambda L_{x}^{n}+S_{n}\right)^{-1} y .
$$

We now give convergence conditions for the finite-dimensional operators $\left\{Q_{n}\right\}$.

Theorem 4.1. Suppose that $X$ has a Schauder basis $\left\{e_{k}\right\}$. on

(A) If $L: X^{k} \mapsto X$ is compact in the $k$ th variable, then $Q_{n}$ converges to $Q$ pointwise

$$
\{x \in X \mid\|\lambda L x\|<1\} \text {. }
$$

(B) If $L: X^{k} \mapsto X$ is compact and $\delta<1$, then $Q_{n}$ converges to $Q$ uniformly on

$$
U_{\delta}=\left\{x \in X \mid\|\lambda L\|\|x\|^{k-1} \leq \delta\right\} .
$$

Proof. Let $x \in\left\{x \in X:\left\|\lambda L_{x}\right\|<1\right\}$ and observe that

$$
\begin{aligned}
Q(x)-Q_{n}(x)= & \left(\lambda L_{x}+I\right)^{-1} y-\left(\lambda L_{x}^{n}+S_{n}\right)^{-1} S_{n}(y) \\
= & \left(\lambda L_{x}+I\right)^{-1}\left(y-S_{n}(y)\right) \\
& +\left(\lambda L_{x}+I\right)^{-1}\left(\left(\lambda L_{x}^{n}+S_{n}\right)-\left(\lambda L_{x}+I\right)\right)\left(\lambda L_{x}^{n}+S_{n}\right)^{-1} S_{n}(y) .
\end{aligned}
$$

Since $\left(\lambda L_{x}^{n}+S_{n}\right)^{-1} S_{n}(y) \in V_{n}$, we have

$$
\left(S_{n}-I\right)\left(\lambda L_{x}^{n}+S_{n}\right)^{-1} S_{n}(y)=0 .
$$

Thus

$$
\begin{aligned}
Q(x)-Q_{n}(x)= & \left(\lambda L_{x}+I\right)^{-1}\left(y-S_{n}(y)\right) \\
& +\left(\lambda L_{x}+I\right)^{-1}\left(\lambda L_{x}^{n}-\lambda L_{x}\right)\left(\lambda L_{x}^{n}+S_{n}\right)^{-1} S_{n}(y)
\end{aligned}
$$

which yields

$$
\begin{aligned}
\| Q(x) & -Q_{n}(x) \| \\
\leq & \frac{1}{1-\left\|\lambda L_{x}\right\|}\left(\left\|y-S_{n}(y)\right\|+\left\|\lambda L_{x}^{n}-\lambda L_{x}\right\|\left\|\left(\lambda L_{x}^{n}+S_{n}\right)^{-1} S_{n}(y)\right\|\right) \\
& \leq \frac{\left\|y-S_{n}(y)\right\|}{1-\left\|\lambda L_{x}\right\|}+\frac{\left\|\lambda L_{x}^{n}-\lambda L_{x}\right\|\|y\|}{\left(1-\left\|\lambda L_{x}\right\|\right)^{2}}
\end{aligned}
$$


To prove assertion (A), we note that for each $x \in X, L_{x}$ is a compact linear map. Now $\left\|\lambda L_{x}^{n}-\lambda L_{x}\right\| \rightarrow 0$ by Lemma $1.2 /(\mathrm{i})$ so that $\left\|Q(x)-Q_{n}(x)\right\| \rightarrow 0$ thus proving (A).

Next assume that $L$ is compact and that $x \in U_{\delta}$. Then

$$
\left\|Q(x)-Q_{n}(x)\right\| \leq \frac{\left\|y-S_{n}(y)\right\|}{1-\delta}+\frac{\left\|\lambda L^{n}-\lambda L\right\|\|y\|}{(1-\delta)^{2}} \frac{\delta}{\|\lambda L\|},
$$

and since $\left\|\lambda L^{n}-\lambda L\right\| \rightarrow 0$ uniformly on $U_{\delta}$ by Lemma $1.2 /(\mathrm{ii}), Q_{n}$ converges to $Q$ uniformly on $U_{\delta}$. This proves assertion (B)

Remark. For the sake of notation, we have chosen $L$ to be compact in the $k$ th variable. The result holds as long as $L$ is compact in at least one variable.

We now state and prove our main result.

Theorem 4.2. Suppose that $X$ has a Schauder basis $\left\{e_{k}\right\}$ and that

Then:

$$
0<|\lambda|\|L\|\|y\|^{k-1}=\alpha<\frac{(k-1)^{k-1}}{k^{k}} .
$$

(a) If $L$ is compact in at least one variable, then $\left\{U_{j}, Q_{j}\right\}$ is a uniformly contractive system.

(b) If $L$ is compact, then the iterative scheme (29) given in Theorem 3.4 converges.

Proof. The first part of the proof is needed for both assertions (a) and (b). Choose $K \in \mathbb{N}$ so that if $j \geq K$, then

$$
\left\|S_{j}(y)-y\right\|+\frac{\alpha(1+D)^{k-1}}{1-\alpha(1+D)^{k-1}} \leq D
$$

where the existence of $D$ is guaranteed by Lemma 2.1. Consider the closed ball

$$
U=\{z \in X \mid\|z-y\| \leq D\|y\|\} \text {. }
$$

Then, arguing similarly to the proof of Theorem 2.2 , for $x \in U$ and $j \geq K$, we have

$$
\begin{aligned}
\left\|Q_{j}(x)-y\right\| & =\left\|\left(\left(\lambda L_{x}^{j}+S_{j}\right)^{-1}-S_{j}\right) y+\left(S_{j}-I\right) y\right\| \\
& =\left\|\left(\lambda L_{x}^{j}+S_{j}\right)^{-1}\left(S_{j}-\left(\lambda L_{x}^{j}+S_{j}\right)\right) y+\left(S_{j}-I\right) y\right\| \\
& \leq \frac{\left\|\lambda L_{x}\right\|}{1-\left\|\lambda L_{x}\right\|}\|y\|+\left\|\left(S_{j}-I\right) y\right\| \\
& \leq \frac{\alpha(1+D)^{k-1}}{1-\alpha(1+D)^{k-1}}\|y\|+\left\|\left(S_{j}-I\right) y\right\| \\
& \leq D\|y\| .
\end{aligned}
$$

Hence $Q_{j}(U) \subset U_{j}$. The proof that each $Q_{j}$ is a contractive map with the same contraction factor $r$ as $Q$ is very similar to that given for $Q$ in the proof of Theorem 2.2 and is omitted. Thus condition (C1) is satisfied.

To complete the proof of part (a), note that $U \subset\left\{x \in X \mid\left\|\lambda L_{x}\right\|<1\right\}$ so Theorem $4.1 /(\mathrm{A})$ applies. Then by Theorem 3.2 , condition (C2) is satisfied. If $L$ is compact, then $Q_{n}$ converges to $Q$ uniformly on $U$ by Theorem 4.1/(B). Therefore Theorem 3.4 applies 
We consider the case $k=2$ so that Theorem 4.2 applies to the quadratic equation

$$
y=x+\lambda B(x, x)
$$

where $B$ is bilinear. We state Theorem 7 of $[1]$ for comparison purposes.

Theorem 4.3 (see Argyros [1: Theorem 7]). Consider the quadratic equations

$$
z=y+F_{n}(z, z)
$$

where $F_{n}: X \times X \rightarrow X \quad(n \in \mathbb{N})$ are bounded symmetric bilinear operators. If

(i) the sequence $\left\{F_{n}\right\}$ converges to $B$ uniformly as $n \rightarrow \infty$

(ii) for each $n$ there exists $z_{n}$, satisfying (34) and $\sup _{n}\left\|z_{n}\right\|<(2|\lambda|\|B\|)^{-1}$, then the sequence $\left\{z_{n}\right\}$ converges to a solution $z$ of equation (33).

Observe that Argyros requires uniform convergence of the operators $F_{n}$ to $B$, while our Theorem $4.2 /($ a) assumes only that $B$ is compact in one variable in order to guarantee that the fixed point solutions $z_{n}$ converge to the solution $z_{s}$ of equation (33).

Also note that Argyros' theorem requires that the bilinear operators $F_{n}$ be symmetric, while this is not needed for Theorem 4.2/(a). This fact is quite important for an operator $B(x, w)$ that is compact in only one variable, for if $B$ is "symmetrized" using the formula

$$
\bar{B}(x, w)=\frac{B(x, w)+B(w, x)}{2}
$$

then the compactness in one variable is destroyed. These points should be kept in mind for Example 5.1 below.

\section{Applications to integral equations}

In the final section of this paper, we employ our iterative scheme and our previous results to obtain approximate solutions to the two classes of integral equations given in the introduction. In both examples, we work in $X=L^{2}[a, b],-\infty \leq a<b \leq \infty$. While we have considered different finite-dimensional subspaces of $L^{2}(\mathbb{R})$ in our examples, we have found that it is quite beneficial to utilize the closed subspaces $V_{n} \subset L^{2}[a, b]$ with $V_{n} \subset V_{n+1}(n \in \mathbb{N})$, that form a multiresolution analysis (MRA) of $L^{2}[a, b]$ (see $[4,7]$ for a treatment of multiresolution analysis). The multiresolution analysis gives rise to a so-called wavelet basis $\left\{\psi_{n k}\right\}$, where for fixed $n,\left\{\psi_{n k}\right\}_{k}$ forms an orthonormal basis for the space $W_{n}, V_{n+1}:=V_{n} \bigoplus W_{n}$. For $n, k \in \mathbb{Z},\left\{\psi_{n k}\right\}$ forms an orthonormal basis for $L^{2}[a, b]$. For $L^{2}(\mathbb{R})$, Daubechies [8] was the first to construct an orthonormal basis $\left\{\psi_{n k}\right\}$ generated from one compactly supported mother wavelet $\psi$. For $L^{2}[a, b],-\infty<$ $a<b<\infty$, similar constructions can be found in $[5,6,13]$ among others.

It is desirable to use an orthonormal basis in the subsequent computations since each iterative step involves solving a linear finite-dimensional system. In addition, the compact support properties of the wavelet basis greatly reduce the number of numerical integrations that must be performed when we project our operator into finite-dimensional subspaces. 
Example 5.1. Consider the Chandrasekhar integral equation

$$
1=H(s)+\frac{1}{2} \lambda s H(s) \int_{0}^{1} \frac{H(t)}{s+t} d t
$$

which arises in the theory of radiative transfer [3].

We consider solving (35) in $L^{2}[0,1]$ for the function $H(s)$. While this equation is generally solved in $C[0,1]$ (see $[1,16])$, it has been shown in [19] that an $L^{2}$-solution to equation (35) is in fact in $C[0,1]$. If we put $y(s)=1$ and define $B: L^{2}[0,1] \times L^{2}[0,1] \mapsto$ $L^{2}[0,1]$ by

$$
B(G, H)(s)=\frac{1}{2} s G(s) \int_{0}^{1} \frac{H(t)}{s+t} d t
$$

then the Chandrasekhar equation (35) can be expressed in the form

$$
y=x+\lambda B(x, x)
$$

To obtain a bound on $\|B\|$, note that

$$
\begin{aligned}
\|B(G, H)\|^{2} & =\frac{1}{4} \int_{0}^{1}\left(s G(s) \int_{0}^{1} \frac{H(t)}{s+t} d t\right)^{2} d s \\
& \leq \frac{1}{4} \int_{0}^{1}|G(s)|^{2}\left(\int_{0}^{1}\left(\frac{s}{s+t}\right)^{2} d t \int_{0}^{1}(H(t))^{2} d t\right) d s
\end{aligned}
$$

by the Cauchy-Schwarz inequality. Then

$$
\|B(G, H)\|^{2} \leq \frac{1}{4} c^{2}\|G\|^{2}\|H\|^{2} \quad \text { where } \quad c^{2}=\sup _{0 \leq s \leq 1} \int_{0}^{1}\left(\frac{s}{s+t}\right)^{2} d t=\frac{1}{2}
$$

so $\|B\| \leq \frac{1}{2 \sqrt{2}}$. Note that the linear operator

$$
B(G, \cdot)(s)=s G(s) \int_{0}^{1} \frac{\cdot}{s+t} d t
$$

is compact for each $G(s) \in L^{2}[0,1]$. Hence Theorem $4.2 /(\mathrm{a})$ applies for appropriate $\lambda$ and $U$, and any orthonormal basis of $L^{2}[0,1]$. It is worthwhile to note that Theorem $4.1 /(B)$ does not apply, since the operator $B$ is only compact in one variable. It is not clear that Theorem 4.3 of Argyros can be applied to this example since $B$ is only compact in one variable. 
This example is an illustration of and not a rationale for Theorem 4.2. Using more information about equation (35) than is used here, it can be proven that (see [3]) equation (35) has a solution for $0 \leq \lambda \leq 1$, while our results apply for $|\lambda| \leq \frac{1}{\sqrt{2}}$. Our numerical experiments indicate that this iterative scheme converges for $0 \leq \lambda \leq 1$, but we choose a $\lambda$ value below that is justified by Theorem $4.2 /(\mathrm{a})$.

In order to compute approximate solutions to eqaation (37), we use the iterative scheme similar to (4):

$$
Q_{n}\left(x^{k}\right)=x^{k+1} \quad(k \in \mathbb{N})
$$

where $Q_{n}$ was defined by (32), and approximate the fixed point $z_{n}$ of (38). We then choose larger and larger $V_{n}$ spaces and repeat the iterative process. Finally, we appeal to Theorem 3.3 to conclude that the $z_{n}$ approach the true solution.

Suppose we wish to compute the fixed point $z_{n}$ of (38). Let $\left\{e_{1}, \ldots, e_{N}\right\}$ be an orthonormal basis for $V_{n}$, set $y(t)=1$, and consider projecting (37) into $V_{n}$

$$
\left(S_{n}+\lambda B_{x^{k}}^{n}\right) x^{k+1}=y^{n}
$$

where $y^{n}(t)=\sum_{i=1}^{N} y_{i}^{n} e_{i}(t)$ with $y_{i}^{n}=\left\langle y, e_{i}\right\rangle$. To obtain a matrix representation $A^{n}$ for $B_{x^{k}}^{n}$, we let

$$
b_{i j}^{k}=\frac{1}{2} \int_{0}^{1} \int_{0}^{1} e_{k}(s) \dot{e}_{i}(s) e_{j}(t) \frac{s}{s+t} d t d s \quad(i, j, k=1, \ldots, N)
$$

so that the entries of $A^{n}$ are given by

$$
a_{p q}=\sum_{i=1}^{N} b_{i q}^{p} x_{i}^{k} \quad \text { where } \quad x^{k}(t)=\sum_{i=1}^{N} x_{i}^{k} e_{i}(t) .
$$

We start with $x^{1}:=y$ and then iterate by repeatedly solving the system

$$
\left[\begin{array}{c}
y_{1}^{n} \\
\vdots \\
y_{N}^{n}
\end{array}\right]=\left(I_{n}+\lambda A^{n}\right)\left[\begin{array}{c}
x_{1}^{k+1} \\
\vdots \\
x_{N}^{k+1}
\end{array}\right]
$$

where the $n \times n$ identity matrix $I_{n}$ is the matrix representation of $S_{n}$. Note that the $b_{i j}^{k}$ 's must only be computed once in this scheme and that $b_{i j}^{k}$ is symmetric in $i$ and $k$. In addition, if the basis functions have compact support within $[0,1]$, then it is possible to a priori assign certain $b_{i j}^{k}=0$. Certain wavelet bases have this property.

To illustrate the above scheme, we have chosen the first two bases to be $N=$ 4 dimensional subspaces while the third choice is an $N=5$ dimensional subspace. We make no claim as to which is the best choice - we provide these three bases for comparative purposes. Figure 1 illustrates our approximate solutions, where $P_{3}([0,1])$ 
is the space of polynomials of degree three or less spanned by the classical Legendre polynomials.

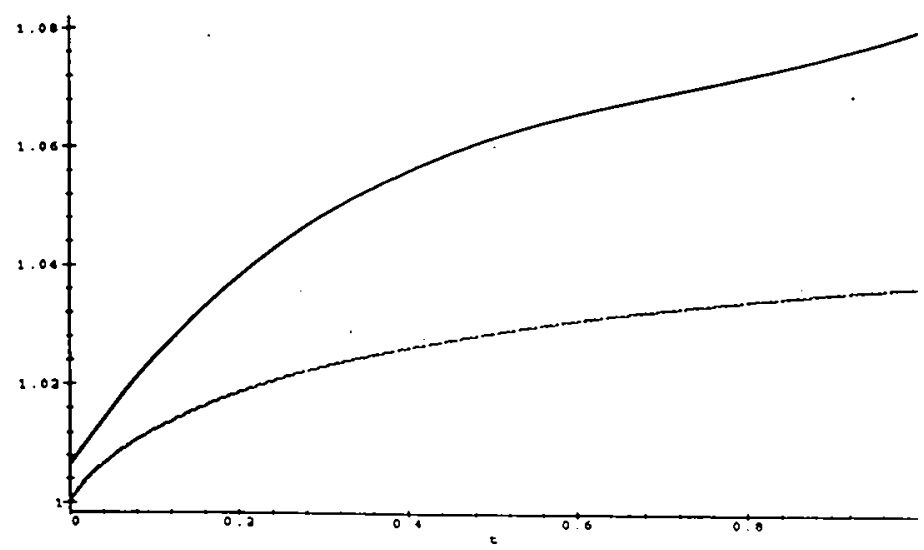

Figure 1: $H(t)$ (solid) and the solution (dotted) from $P_{3}([0,1])$

Further, let $S_{1}^{2}([0,1])$ be the space of piecewise continuously differentiable quadratic polynomials with possible breakpoints at $0, \frac{1}{2}$ and 1 , spanned by orthonormalized $B$ splines. These splines serve as a basis for a $V_{1}([0,1])$ space given in Chui and Quak [5]. The results of our computations are given in Figure 2. In each case, $\lambda=.1$ and six iterations were performed.

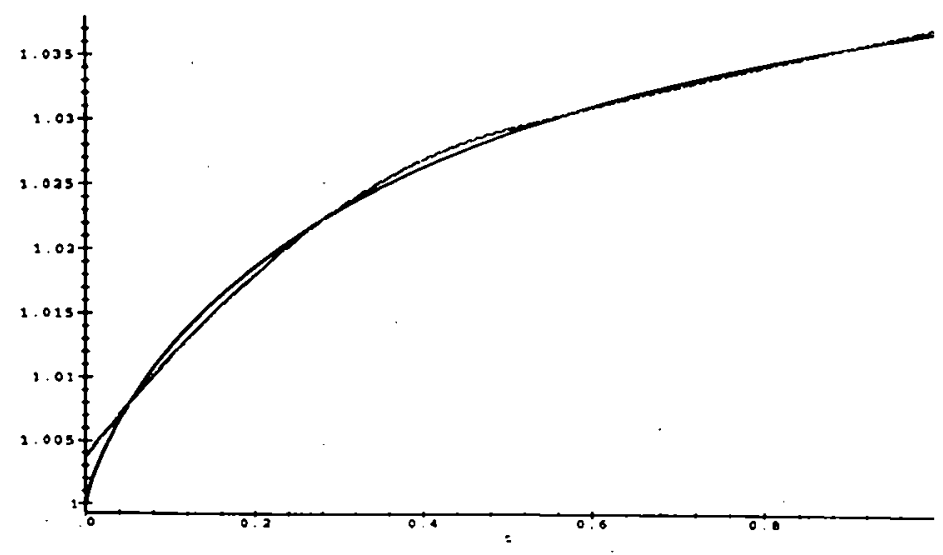

Figure 2: $H(t)$ (solid) and the solution (dotted) from $S_{1}^{2}([0,1])$

Finally, let $S_{0}^{1}([0,1])$ the space of continuous linear polynomials with possible breakpoints at $0, \frac{1}{4}, \frac{1}{2}, \frac{3}{4}$ and 1 , spanned by orthonormalized $B$-splines. These splines serve 
as a basis for a $V_{1}([0,1])$ space given in [5]. The results of our computations are given in Figure 3. Also here, in each case, $\lambda=.1$ and six iterations were performed.

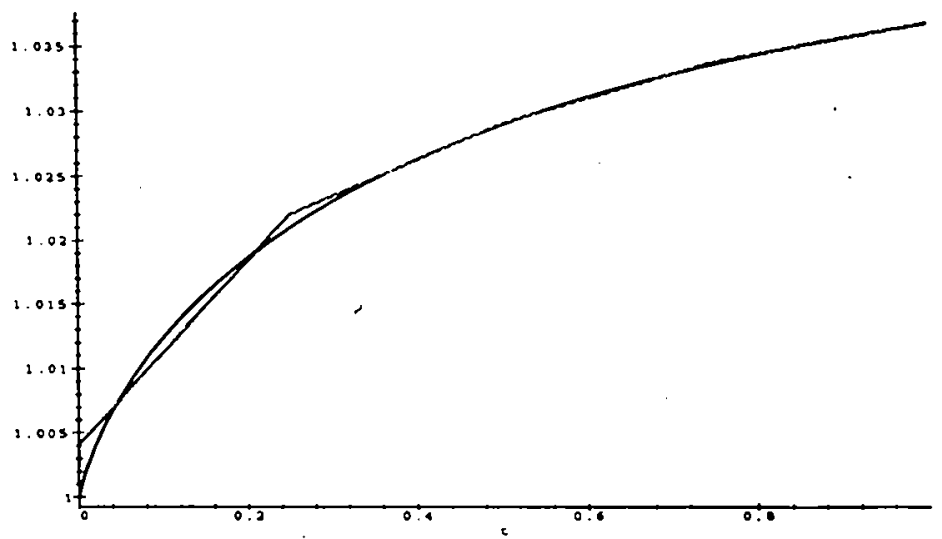

Figure 3: $H(t)$ (solid) and the solution (dotted) from $S_{0}^{2}([0,1])$

Remark. The spaces $S_{1}^{2}([0,1])$ and $S_{0}^{2}([0,1])$ can be viewed as generated by scaling functions and these functions are compactly supported. Wavelet analysis allows us to readily move to the next larger space using the identity $V_{n+1}=V_{n} \bigoplus W_{n}$. As $n$ grows, the support of both the scaling functions and the wavelets shrink thus reducing the number of numerical integrations that need to be computed.

The exact solution to equation (35) is given in [16] and is of the form

$$
H(t)=\exp \left(-\frac{t}{\pi} \int_{0}^{\pi / 2} \frac{\log (1-\lambda s \cot s)}{\cos ^{2} s+t^{2} \sin ^{2} s} d s\right) .
$$

Rall (16) used a different iterative procedure. He computed some values

\begin{tabular}{c|c|c|c|c}
$t$ & Rall & $P_{3}([0,1])$ & $S_{1}^{2}([0,1])$ & $S_{0}^{1}([0,1])$ \\
\hline 0.0159199 & 1.00333256 & 1.00958383 & 1.00506878 & 1.00519491 \\
0.0819844 & 1.01089700 & 1.02134388 & 1.01025220 & 1.00994962 \\
0.1933143 & 1.01829896 & 1.03723329 & 1.01764377 & 1.01796209 \\
0.3378733 & 1.02435469 & 1.05173649 & 1.02472538 & 1.02450330 \\
0.5000000 & 1.02892234 & 1.06190891 & 1.02928548 & 1.02904480 \\
0.6621267 & 1.03220522 & 1.06818221 & 1.03198365 & 1.03211930 \\
0.8066857 & 1.03445865 & 1.07263226 & 1.03426463 & 1.03448647 \\
0.9180156 & 1.03589121 & 1.07655348 & 1.03594109 & 1.03586278 \\
0.9840801 & 1.03664375 & 1.07947299 & 1.03690293 & 1.03667950
\end{tabular}

of his approximate solution in a ten-dimensional subspace of $C([0,1])$ and his computa- 
tions used seven iterates. For comparison purposes, we list his values with values from our approximate solutions in the table above.

The solution to the Chandrashekhar equation (35) is usually approximated in $C[0,1]$ rather than $L^{2}[0,1]$ (see $\left.[1,17]\right)$. Theorem 4.2 is general enough to be applied to $C[0,1]$ since only a Schauder basis is assumed. A wavelet basis for a dense subspace of $C[0,1]$ has been reported by Wang [21], so that the benefits of wavelets can be retained if an approximate solution in $C[0,1]$ is desired.

Example 5.2. Solve for $x \in L^{2}[a, b]$ the Hammerstein equation

$$
y(s)=x(s)+\lambda \int_{a}^{b} k(s, t)(x(t))^{2} d t
$$

This equation can be expressed as a bilinear one in $L^{2}[a, b]$ where

$$
K\left(f_{1}, f_{2}\right)(s)=\int_{a}^{b} k(s, t) f_{1}(t) f_{2}(t) d t
$$

defines a bilinear operator $K: L^{2}[a, b] \times L^{2}[a, b] \mapsto L^{2}[a, b]$. Using the Cauchy-Schwarz inequality, the map $K$ can be shown to be bounded if

$$
k^{*}(s)=\sup _{a \leq t \leq b}|k(s, t)| \in L^{2}[a, b] .
$$

The linear operator

$$
K\left(f_{1}, \cdot\right)(s)=\int_{a}^{b} k(s, t) f_{1}(t)(\cdot) d t
$$

is compact for each $f_{1} \in L^{2}[a, b]$ when (41) holds. Thus Theorem $4.2 /(a)$ applies. Actually, we can claim that Theorem $4.2 /(\mathrm{b})$ applies, since $K$ is actually compact (see [18]).

We have used the iterative scheme (29). This method is quite similar to that used in Example 5.1 only now each successive iteration takes place in a larger subspace. In the case when $X=L^{2}(\mathbb{R})$, it is quite convenient to employ the ladder of subspaces given in Daubechies [7].

Example 5.3. Consider the Hammerstein integral equation

$$
y(s)=x(s)+\lambda \int_{a}^{b} k\left(s, t_{1}, t_{2}, \ldots, t_{n}\right) x\left(t_{1}\right) \cdots x\left(t_{n}\right) d t_{1} \cdots d t_{n} .
$$

As in Example 5.2, (42) can also be viewed as an $n$-linear equation in $L^{2}[a, b]$

$$
y=x+\lambda L(x, \ldots, x)
$$


where the $n$-linear operator

$$
L: L^{2}[a, b] \times L^{2}[a, b] \times \cdots \times L^{2}[a, b] \mapsto L^{2}[a, b]
$$

is defined by

$$
L\left(f_{1}, f_{2}, \ldots, f_{n}\right)(s)=\int_{a}^{b} k\left(s, t_{1}, t_{2}, \ldots, t_{n}\right) f_{1}\left(t_{1}\right) f_{2}\left(t_{2}\right) \cdots f_{n}\left(t_{n}\right) d t_{1} d t_{2} \cdots d t_{n} .
$$

It can be shown (see [20]) that $L$ is in fact compact when the kernel function is in $L^{2}\left([a, b]^{n+1}\right)$, so Theorem $4.2 /(\mathrm{b})$ and the iterative scheme (29) apply.

Acknowledgement. The authors wish to thank the anonymous referees for their constructive comments. In particular we wish to thank one of the referees for remarks leading to Proposition 2.6.

\section{References}

[1] Argyros, I. K.: Quadratic equations and applications to Chandrashekhar's and related equations. Bull. Austral. Math. Soc. 32 (1985), 275 - 292.

[2] Argyros, I. K. and F. Szidarovszsky: The Theory and Applications of Iteration Methods. Boca Raton (Florida, USA): CRC Press 1993.

[3] Chandrashekhar, S.: Radiative Transfer. New York: Dover 1960.

[4] Chui, C. K.: An Introduction to Wavelets. San Diego Acad. Press 1992.

[5] Chui, C. K. and E. Quak: Wavelets on a bounded interval. In: Numerical Methods of Approximation Theory, Vol. 9 (eds.: D. Braess and Larry L. Schumaker; Intern. Ser. Num. Math.: Vol. 105). Basel: Birkhäuser Verlag 1992, pp. 53 - 75.

[6] Cohen, A., Daubechies, I. and P. Vial: Wavelets on the interval and fast wavelet transforms. Appl. Comp. Harmonic Anal. 1 (1993), 54 - 81.

[7] Daubechies, I.: Ten Lectures on Wavelets (Conf. Board Math. Sci. (CBMS): Vol. 61). Philadelphia: SIAM 1992.

[8] Daubechies, I.: Orthonormal bases of compactly supported wavelets. Comm. Pure Appl. Math. 41 (1988), 909 - 996.

[9] DePree, J. D. and C. W. Swartz: Introduction to Real Analysis. New York: Wiley 1988.

[10] Keller, H. and D. Cohen: Some positone problems suggested by nonlinear heat generation. J. Math. Mech. 16 (1967), 1361 - 1376.

[11] Luning, C. D. and W. L. Perry: Positive solutions of negative exponent generalized EmdenFowler boundary value problems. SIAM J. Math. Anal. 12 (1981), 874 - 879.

[12] McFarland, J. E.: An iterative solution of the quadratic equation in Banach spaces. Proc. Amer. Math. Soc. 12 (1958), $824-830$.

[13) Meyer, Y.: Ondelettes sur l'intervalle. Rev. Mat. Iberoamericana 7 (1991), 115 - 143.

[14] Pietsch, A.: Ideals of multilinear functionals. In: Proc. II Intern. Conf. on Oper. Alg., Ideals, and Their Appl. in Theor. Phys., Leipzig 1983 (eds.: H. Baumgärtel et al; 
Teubner-Texte zur Mathematik: Vol. 67). Leipzig: B. G. Teubner Verlagsges. 1984, pp. $185-199$.

[15] Prenter, P.: On polynomial operators and equations. In: Nonlin. Funct. Anal. App. (ed.: L. B. Rall). New York: Acad. Press 1971, pp. 361 - 398.

[16] Rall, L. B.: Computational Solution of Nonlinear Operator Equations. New York: Wiley 1969.

[17] Rall, L. B.: Quadratic equations in Banach spaces. Rend. Circ. Math. Palermo 10 (1961), $314-332$.

[18] Ruch, D. K.: Characterizations of compact bilinear maps. Lin. Multilin. Alg. 25 (1989), $297-307$.

[19] Ruch, D. K.: On uniformly contractive systems and quadratic equations in Banach space. Bull. Austr. Math. Society 95 (1995) (to appear).

[20] Ruch, D. K.: Completely continuous and related multilinear operators. Rend. Circ. di Palermo 44 (1995) (to appear).

[21] Wang, J. Z.: A cubic spline wavelet basis of Sobolev spaces and multilevel interpolations. Preprint 1995.

Received 21.03.1995; in revised form 03.08.1995 